\title{
Developing Experiential Exhibitions Based on Conservation Science Content of Bronze Mirror
}

\author{
Young Hoon Jo,", Jikio Kim², Yong Hyun Yun ${ }^{3}$, Nam Chul Cho', Chan Hee Lee ${ }^{1}$ \\ ${ }^{I}$ Department of Cultural Heritage Conservation Sciences, Kongju National University, Gongju 32588, Korea \\ ${ }^{2}$ Technology Research Institute for Cultural Heritage, Daejeon 34857, Korea \\ ${ }^{3}$ Science Heritage and Preservation Division, National Science Museum, Daejeon 34143, Korea
}

\author{
Received August 4, 2021 \\ Revised August 10, 2021 \\ Accepted August 12, 2021 \\ *Corresponding author \\ E-mail: joyh@kongju.ac.kr \\ Phone: +82-41-850-8539 \\ Journal of Conservation Science \\ 2021;37(4):362-369 \\ https://doi.org/10.12654/JCS.2021. \\ 37.4 .05 \\ pISSN: 1225-5459, eISSN: 2287-9781 \\ (C) The Korean Society of \\ Conservation Science for Cultural \\ Heritage \\ This is an Open-Access article distributed \\ under the terms of the Creative \\ Commons Attribution Non-Commercial \\ License (http://creativecommons.org/ \\ licenses/by-nc/3.0) which permits \\ unrestricted non-commercial use, \\ distribution, and reproduction in any \\ medium, provided the original work is \\ properly cited.
}

\begin{abstract}
In museums, exhibition content focuses mostly on cultural heritage's historical values and functions, but doing so tends to limit visitors' interest and immersion. To counter this limitation, the study developed an experiential media art exhibition fusing bronze mirrors' traditional production technology and modern conservation science. First, for the exhibition system, scientific cultural heritage contents were projected on the three-dimensional (3D) printed bronze mirror through interactions between motion recognition digital information display (DID) and the projector. Then, a scenario of 17 missions in four stages (production process, corrosion mechanism, scientific analysis and diagnosis, and conservation treatment and restoration) was prepared according to the temporal spectrum. Additionally, various media art effects and interaction technologies were developed, so visitors could understand and become immersed in bronze mirrors' scientific content. A user test was evaluated through the living lab, reflecting generally high levels of satisfaction ( 90.2 points). Qualitative evaluation was generally positive, with comments such as "easy to understand and useful as the esoteric science exhibition was combined with media art" (16.7\%), "wonderful and interesting" (11.7\%), and "firsthand experience was good" (9.2\%). By combining an esoteric science exhibition centered on principles and theories with visual media art and by developing an immersive directing method to provide high-level exhibition technology, the exhibition induced visitors' active participation. This exhibition's content can become an important platform for expanding universal museum exhibitions on archaeology, history, and art into conservation science.
\end{abstract}

Key Words Bronze mirror, Conservation science content, Experiential exhibitions, Media art, Living lab

\section{INTRODUCTION}

Many countries with excellent cultural heritage are actively developing exhibitions of digital cultural content. Such previous digital exhibitions have often been delivered unilaterally using sound and video, but doing so limits visitors' interest and immersion into the content. Moreover, such delivery insufficiently explains cultural heritage's historical value and may lead to decreased attraction and degradation of economic feasibility compared to long-term investment costs.

Recently, along with the ICT industry's rapid development, media art exhibitions are combining cultural heritage with digital technology to counter the unilateral problem described above and to satisfy the demand for content presented through new methods. Unlike previous unilaterally appreciated exhibits, visitors can interact with media art through cultural and artistic works' transformations that enhance their content's diversity. Therefore, history museums, art galleries, and science museums are actively using media art as new exhibition technology (Bai and Boo, 2011; Kuo et al., 2016; Yamazoe et al., 2018).

Media art exhibitions of cultural heritage mainly concentrate on buildings with walls and facades because, as 
targets of projection mapping, they are advantageous in inducing the audience's sense of immersion and can be efficiently produced as video content because they are relatively flat (Krautsack, 2011; Yun et al., 2013; Nofal et al., 2018). On the other hand, movable artifacts are not frequently the targets of media art because they are too small for projection mapping.

Additionally, because exhibition content in history and science museums focuses mostly on cultural heritage's historical values and functions, the range visitors can experience is limited (Jeon, 2016; Seong and Lee, 2017). Therefore, new exhibition services are needed to induce visitors' interest and active participation by expanding humanities cultural heritage content to a scientific viewpoint. In particular, combining media art with high visual effects, so visitors can intuitively understand complex and esoteric scientific principles is very important (Kim et al., 2009; Noh et al., 2011).

This study developed an experiential media art exhibition service based on conservation science content that enables intuitive understanding of the cultural heritage production process, in this case, the corrosion mechanism, scientific analysis and diagnosis, conservation treatment, and restoration through interactive projection mapping technology. A bronze mirror with a fine linear design that has many scientific properties was selected as the object of study. The core content is the convergence of traditional science as it relates to the bronze mirror's production and modern conservation science that restores the corroded mirror's function. In addition, interactive projection mapping technology was applied to a three-dimensional (3D) printed bronze mirror for realization of media art.

\section{OBJECT OF CONTENT}

As scientific cultural heritage, the bronze mirror contains convergent elements for media art exhibitions in science, technology, engineering, mathematics, and art. In particular, Korea's National Treasure No. 141, a unique bronze mirror with a fine linear design, has excellent reflectance and clarity comparable to modern mirrors: With a diameter of approximately $21 \mathrm{~cm}$, it has about 13,000 straight lines and 100 concentric circles drawn on it. Its production technique constitutes a mysterious legacy because even in modern

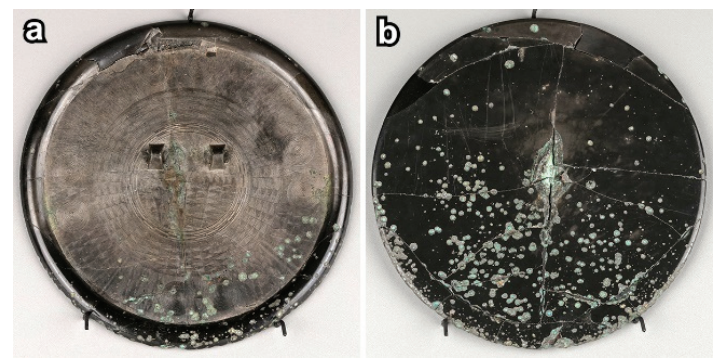

Figure 1. Bronze mirror with a fine linear design in the Korean Christian Museum at Soongsil University (National Treasure No. 141).

times, it has not been reproducible (Figure 1).

However, since most bronze mirrors now in museums were deformed in color and shape due to corrosion and are mainly exhibited for their patterned faces, in many cases, visitors do not recognize their uses and functions (Jeon et al., 2008). Therefore, developing scenarios based on traditional production technology and modern conservation science that restores bronze mirrors' original form is highly appropriate creative content.

In this study, data on history, art, and science related to bronze mirrors were collected to enhance the reliability of the exhibition's content. Based on the data, a scenario of 17 missions in four stages was prepared according to the temporal spectrum. In particular, various media art effects and interaction technologies were developed so users can understand and become immersed in convergent elements, that is, in the science, technology, engineering, mathematics, and art of bronze mirrors.

\section{CONSTRUCTION OF AN EXHIBITION SYSTEM}

Most importantly for construction of the bronze mirror exhibition, a multisensory exhibition had to connect virtuality and reality while inducing a sense of immersion in the content through user interactions. Thus, interactive projection mapping technology was selected to realize the conservation science content of bronze mirrors as media art (Grundhöfer and Iwai, 2018; Guo et al., 2018). This technology is the optimal method for helping audiences intuitively understand bronze mirrors' traditional technology and modern conservation science by interacting with the user's recognition of motion according to the content utilization method (Figure 2a, b). 

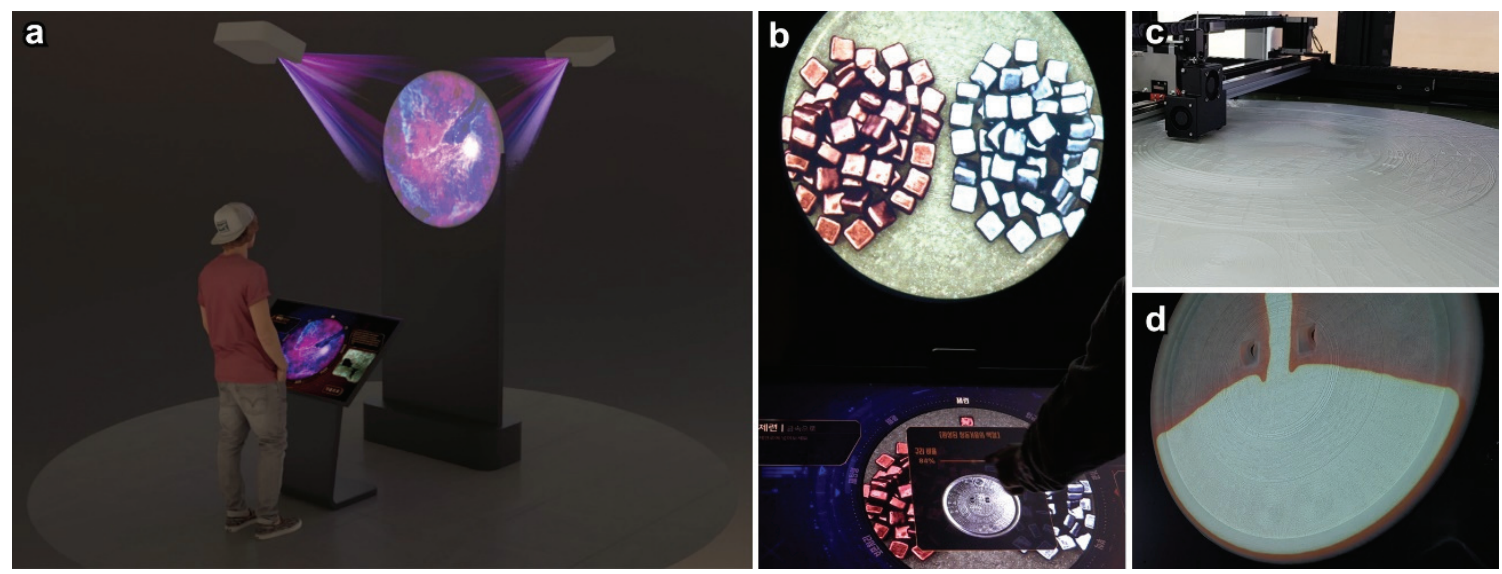

Figure 2. Exhibition system based on the interactive projection mapping. (a) Conceptual rendering image. (b) Real exhibition system. (c) The 3D printing output using material extrusion. (d) The 3D printed bronze mirror for projection mapping.

In addition, at the center of the exhibition space, a large (approx. $1 \mathrm{~m}$ ), 3D, printed bronze mirror was installed so users and visitors can immerse themselves in scientific cultural heritage content (Figure 2c). The 3D printing output method was material extrusion using PLA. In particular, content was projected on the printed bronze mirror through interactions between the motion recognition digital information display (DID) and the projector. That is, when the user's motion instructions are input through DID guidance, special effects are expressed along with various content, and the user experiences various stage-by-stage "missions" related to traditional technology and modern conservation science (Figure 2d). Specifically, projection mapping was simultaneously performed on the printed bronze mirror's front and back to increase the user's immersive sense and the stereognostic effect.

\section{DEVELOPMENT OF AN INTERACTIVE MEDIA ART SCENARIO}

\subsection{Production of bronze mirror (Stage 1)}
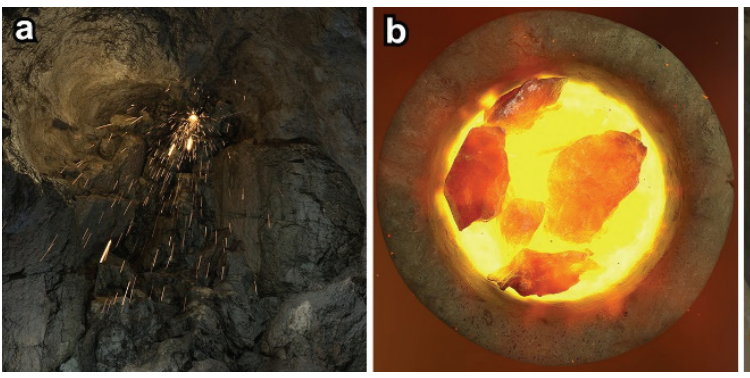

Figure 3. "Production of bronze mirror" stage. (a) Mining.
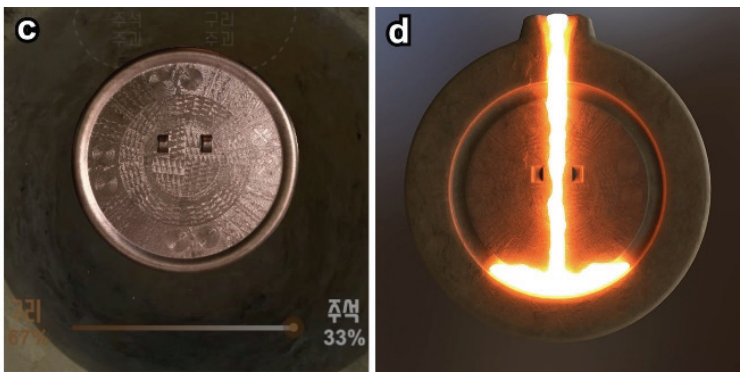

First, in the "production of bronze mirror" stage, the user experiences traditional scientific technologies for producing bronze mirrors, for example, mining, smelting, alloying, casting, and polishing. During mining (Mission 1), the user is immersed in the mining environment to obtain raw material for a bronze mirror (Figure 3a). When the user taps the ore displayed in the kiosk, sound and flame effects are generated. Next, the user experiences smelting (Mission 2) during which ore is turned into metal (Figure 3b). The user learns that the bronze mirror's color changes as the alloy ratio (Mission 3) is adjusted between copper and tin ingots (Figure 3c). Then, the user completes the bronze mirror through the casting process (Mission 4) in which melted metal is poured into the mirror mold to cool (Figure 3d). Finally, when the user selects a piece of leather and polishes the bronze surface, a bronze mirror with a reflective effect is completed (Mission 5). Furthermore, through the camera's mirror function, an effect that enables a user to understand the mirror function was added to the exhibition.

(b) Smelting. (c) Alloying. (d) Casting. 


\subsection{Corrosion and discovery of the bronze mirror} (Stage 2)

In the "corrosion and discovery of the bronze mirror" stage, a user comes to understand a corroded bronze mirror's appearance. Because of exposure to burial environments for at least thousands of years, when newly discovered, bronze mirrors are chemically corroded. In the corrosion stage, media art's visual effects actively enhance the user's learning about the somewhat difficult chemical process. When the user controls the time spectrum - from the prehistoric to the present- first, the bronze mirror is buried in the ground (Figure 4a) and corrosion begins (Mission 6). The user is immersed in the molecular structure's micro-environment, and at this time, oxygen, moisture (Figure $4 b$ ), and chlorides (Figure $4 \mathrm{c}$ ) become attached to the mirror and cause the bronze disease (Mission 7). Next, to maximize the educational effect, the bronze mirror, which has lost its mirror function and become discolored (black), is shown through excavation (Mission 8).

\subsection{Conservation scientist's eye (Stage 3)}

In the "conservation scientist's eye" stage, the user examines the corroded bronze mirror from a scientific viewpoint, prescribing restoration of its original shape. Therefore, the user actively participates in the five missions of recording, observation, analysis, diagnosis, and prescription, while surrounding visitors can appreciate content projected onto the printed bronze mirror. First, during recording (Mission 9), a corroded and damaged bronze mirror is precisely modeled through $3 \mathrm{D}$ scanning (Figure $5 \mathrm{a}$ ). Next, during observation (Mission 10), the corrosive is microscopically observed to determine the bronze disease's cause (Figure 5b). During analysis (Mission 11), the bronze mirror's corrosive and components are identified using an X-ray fluorescence analyzer (Figure 5c). Then, during diagnosis (Mission 12), the mirror's condition is understood through X-ray radiography (Figure 5d). Last, during prescription (Mission 13), through active effort, the user documents data and takes them.
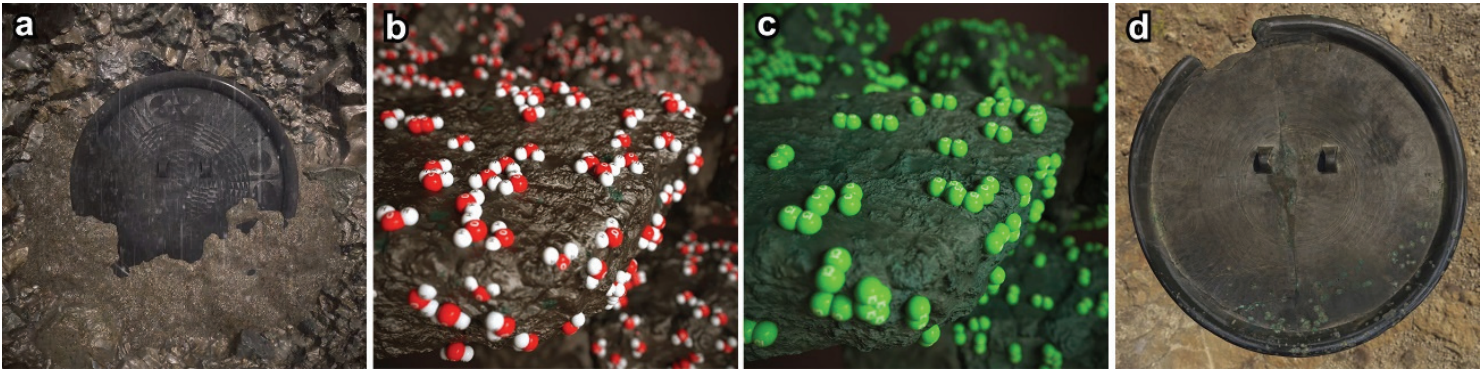

Figure 4. "Corrosion and discovery of the bronze mirror" stage. (a) The bronze mirror buried in the ground. (b, c) User immersed in the corrosion environment. (d) Discovery of the corroded bronze mirror through excavation.
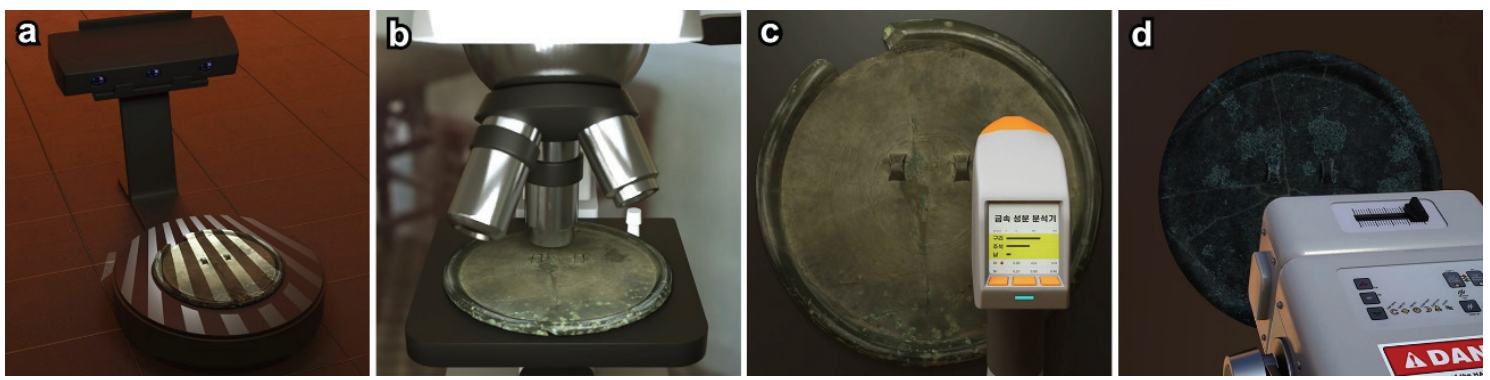

Figure 5. "Conservation scientist's eye" stage. (a) Modeling and recording through 3D scanning. (b) Observation of the bronze disease's cause. (c) Identification of the bronze mirror's corrosive and components using an X-ray fluorescence analyzer. (d) Diagnosis of mirror's condition through X-ray radiography. 

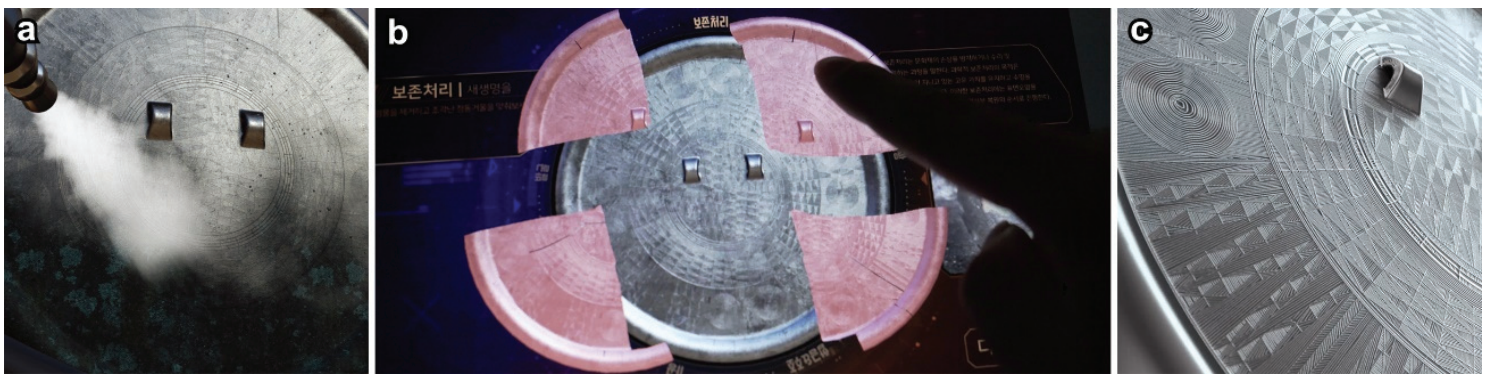

Figure 6. "Giving new life to the bronze mirror" stage. (a) Removal of the corrosive on the mirror's surface. (b) Joining of separated mirror fragments through user interaction. (c) Restored bronze mirror by a user.

\subsection{Giving new life to the bronze mirror (Stage 4)}

In the stage "giving new life to the bronze mirror," the user becomes a conservation scientist by cleaning, strengthening, joining, and restoring the corroded bronze mirror, thereby experiencing the entire restoration process. At stage 4 , the user participates actively through motion recognition interactive technology. First, during cleaning (Mission 14), the user removes the corrosive on the mirror's surface (Figure 6a). Next, to strengthen its weakened physical properties, the surface is impregnated with synthetic resin (Mission 15), and the user joins its separated fragments virtually (Mission 16) (Figure 6b), so the bronze mirror is restored to its original shape (Mission 17) (Figure 6c).

\section{LIVING LAB RESULTS}

Literally a "living laboratory," a living lab solves problems with technologies. A "living lab" refers to an "open" laboratory where, rather than only researchers inside an institutional laboratory, consumers (e.g., users, visitors, operators) participate to solve problems and create results. In other words, the living lab is where consumers and objects

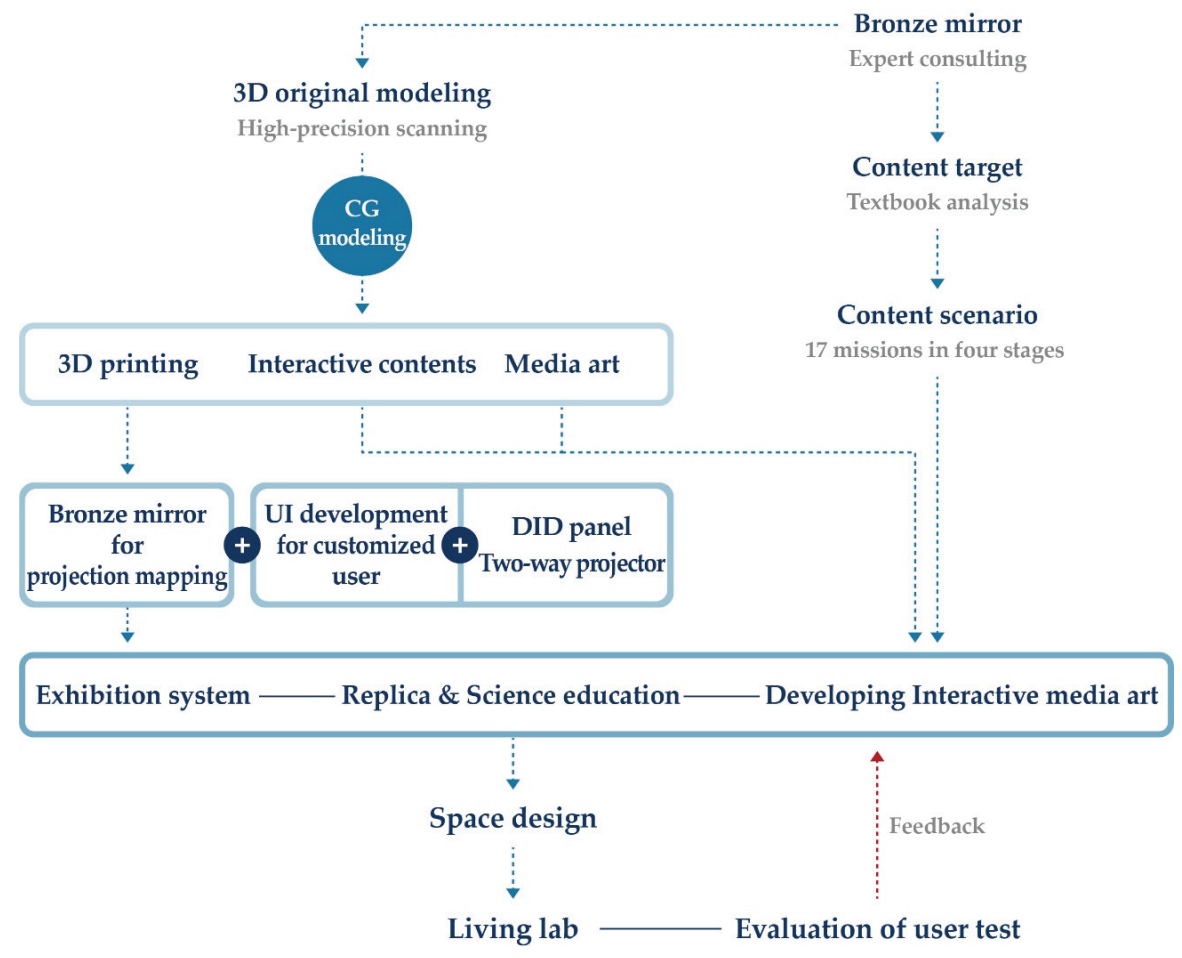

Figure 7. Diagram showing the research performance system. 
of studies exist - not in a research institute or university laboratory. Living labs have recently responded to history and science museums' previous and common installation of exhibits without sufficient verification by examining problems in advance and improving exhibits' quality.

This study implemented a living lab to evaluate media art content's usability and responsiveness in exhibition of the bronze mirror, a scientific cultural heritage. In particular, the study closely analyzed feedback through on-site evaluation by visitors and related experts to improve the exhibition's content (Figure 7). Thus, with the theme of "Bronze Mirror, Reflecting Science," a living lab was held for four days in the first-floor showroom of South Korea's National Science Museum (Figure 8a, b). In the evaluation of user tests, 55 students, 46 public visitors, 11 experts in the Science Museum, and 8 conservation scientists participated. The survey method was field paper assisted personal interviewing using a structured questionnaire, and the survey was conducted with 22 questions about the exhibition itself and user-visitor satisfaction (Figure 9).

As evaluated through the living lab, the exhibit drew very high levels of satisfaction in general ( 90.2 points). By group, conservation scientists and students had high scores, and by age, teenagers and visitors in their 30s had high scores (Table 1). The most helpful method for understanding the exhibit's content was media art videos. Experiencing traditional production technologies and scientific restoration was evaluated as having interesting stages and missions,
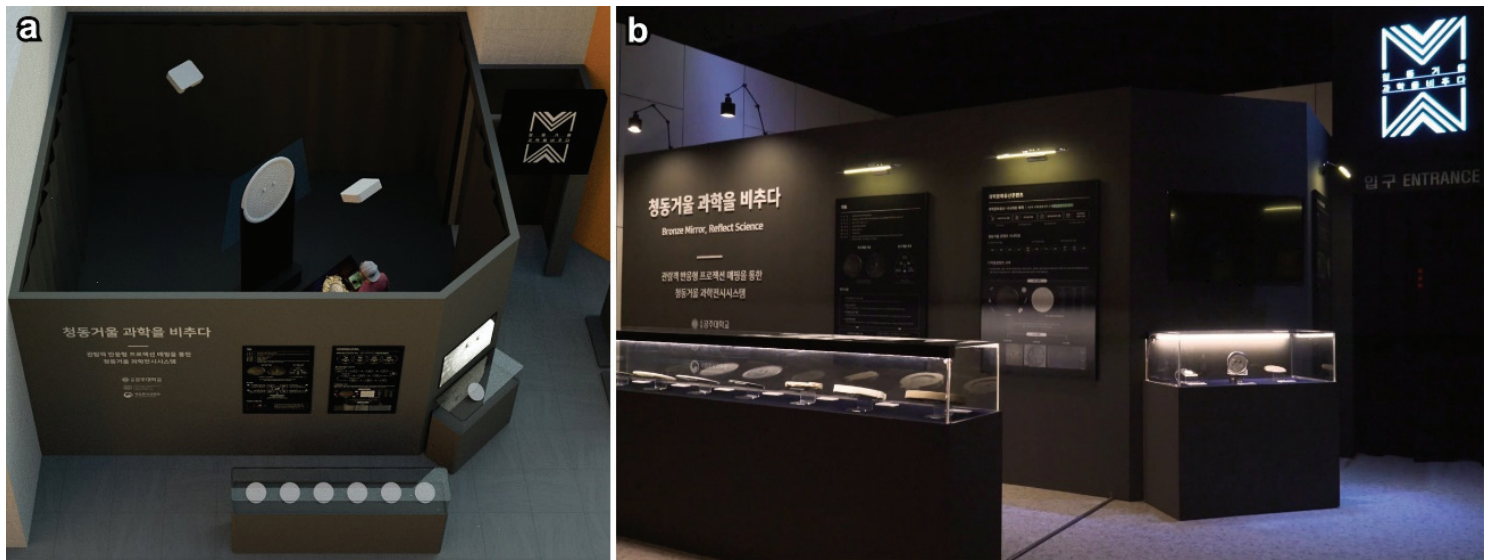

Figure 8. "Bronze Mirror, Reflecting Science" living lab. (a) Stereoscopic image. (b) Real exhibition for the living lab.
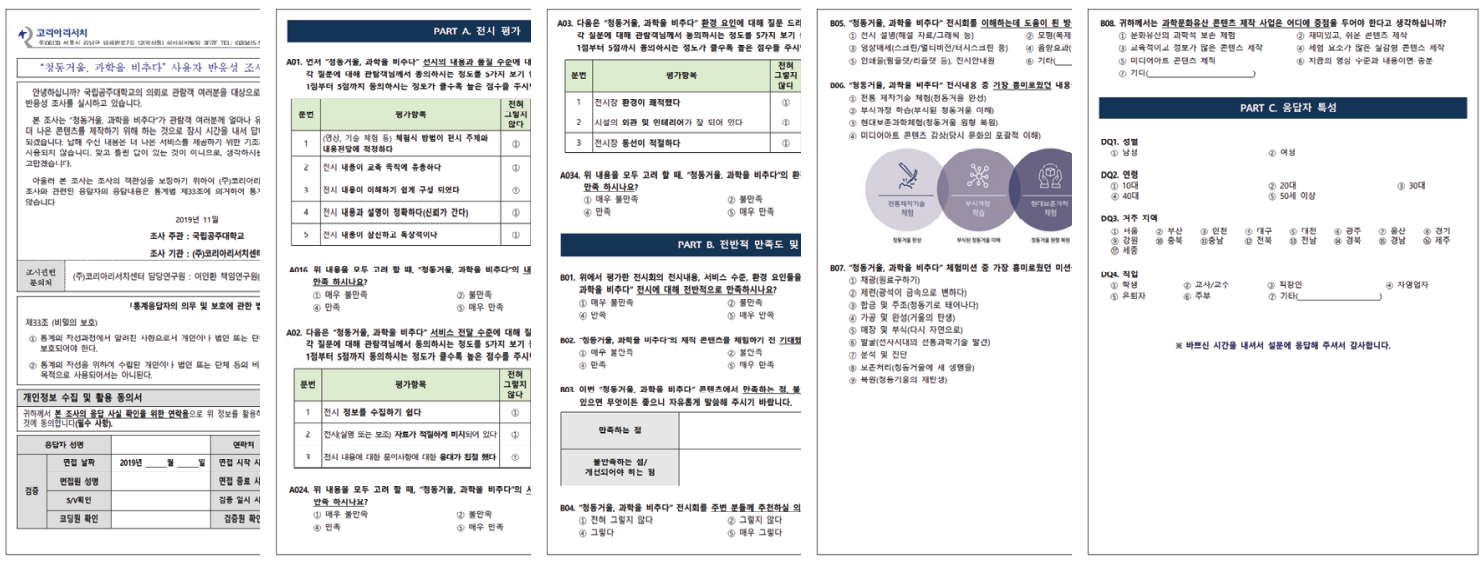

Figure 9. Personal interviewing using a structured questionnaire to evaluate media art content's usability and responsiveness in exhibition of the bronze mirror. 
Table 1. Evaluation of user tests through the living lab

\begin{tabular}{|c|c|c|c|c|c|c|}
\hline & Target users & $\begin{array}{c}\text { Contents \& } \\
\text { quality }\end{array}$ & $\begin{array}{l}\text { Service } \\
\text { delivery }\end{array}$ & $\begin{array}{c}\text { Exhibition } \\
\text { space }\end{array}$ & $\begin{array}{l}\text { Experiential } \\
\text { satisfaction }\end{array}$ & $\begin{array}{c}\text { Overall } \\
\text { satisfaction }\end{array}$ \\
\hline \multirow{4}{*}{ Group } & Students (55) & 90.9 & 92.7 & 90.0 & 90.9 & 91.1 \\
\hline & Public visitors (46) & 88.6 & 88.6 & 88.6 & 89.7 & 88.9 \\
\hline & $\begin{array}{l}\text { Experts in science } \\
\text { museum (11) }\end{array}$ & 90.0 & 88.6 & 84.1 & 88.6 & 87.5 \\
\hline & Conservation scientist (8) & 100.0 & 93.8 & 90.6 & 93.8 & 94.5 \\
\hline \multirow{2}{*}{ Gender } & Male $(60)$ & 91.3 & 92.1 & 89.2 & 91.3 & 90.9 \\
\hline & Female (60) & 89.8 & 89.6 & 88.8 & 89.6 & 89.4 \\
\hline \multirow{6}{*}{ Age } & 10s (38) & 89.5 & 91.4 & 88.2 & 89.5 & 89.6 \\
\hline & $20 \mathrm{~s}(20)$ & 95.0 & 95.0 & 92.5 & 95.0 & 94.4 \\
\hline & 30 s $(22)$ & 92.0 & 92.0 & 88.6 & 94.3 & 91.8 \\
\hline & 40s (18) & 90.3 & 84.7 & 84.7 & 87.5 & 86.8 \\
\hline & 50 s (22) & 86.9 & 89.8 & 90.9 & 86.4 & 88.4 \\
\hline & Average points & 90.5 & 90.8 & 89.0 & 90.4 & 90.2 \\
\hline
\end{tabular}

Values are presented as points.

respectively. As for emphasis in future production, interesting and easy content and experiences of scientific conservation were frequently requested.

The exhibition's qualitative evaluation was generally positive, with comments such as "easy to understand and useful as the esoteric science exhibition was combined with media art" (16.7\%), "wonderful and interesting" (11.7\%), and "firsthand experience was good" (9.2\%). In general, however, the percentage of advice on the media art space (narrow, dark, entrance and exit should be separated) was very high at $19.2 \%$. Furthermore, the need for new interactive and experiential content $(4.2 \%)$ and for supplementation of the content's length and history (3.3\%) was expressed. In the future, rather than operating a living lab in a given space, a space should be designed in which researched and developed exhibition content can be implemented, and the entrance and exit should be separated and the inside brightened.

\section{DISCUSSION AND CONCLUSION}

For this study, exhibition content based on conservation science that enables easy, intuitive understanding of production, corrosion, excavation, diagnosis, analysis, conservation treatment, and restoration of bronze mirrors was constructed. In particular, the active participation of visitors was induced by combining an esoteric science exhibition focused on principles and theories with visual media art. Moreover, an immersive directing method was developed to provide high-level exhibition technology. The exhibition's content is usable as a platform for expanding universal museum exhibitions centered on archaeology, history, and art to the field of conservation science.

So far, the bronze mirror's conservation science content is not complete, but to reflect evaluation of user tests from the living lab, we will supplement it continuously. First, the conservation science scenario's quantitative and qualitative fidelity will be improved, as will interaction methods, including UI and UX, to secure completeness. Because, so far, one-stop content in one system has been realized, DID panel-centered interactions were applied. However, interaction technologies suitable for scenarios by stage should be applied in the future.

To do so, integrated exhibition service platforms should be constructed separately for a "media art screening zone" and "specialized content experience zones by stage." To enhance visitors' immersion and overall understanding of content, the media art screening zone will show bronze mirror conservation science content with projection mapping technology but without any interaction at the exhibition space's center. As for specialized content experience zones by stage, the core scenario's four stages (i.e., production 
process, corrosion mechanism and excavation, analysis and diagnosis, and conservation treatment and restoration) will be separated into individual exhibition systems that enable specialized, deepened experiences.

Since the bronze mirror's conservation science content based on interactive projection mapping is an experiential exhibition system that combines traditional technology, modern conservation science, and futuristic exhibition technology, its value as source technology is very high. In addition, 3D scanning, modeling, and printing technologies were actively used to enhance the exhibition system and media art contents, resulting in a more realistic experience. The conservation science content, exhibition system, advanced technologies, and media art effects provided a new directing method immersed in the cultural heritage unlike previous exhibition contents in the science museum. Since bronze mirrors have excellent global universality, the exhibition's content can be broadly applied to East Asian and European markets, to serve as an important opportunity to popularize Korean scientific cultural heritage and for South Korea to leap into becoming a content-power country.

Because this study focused on whether a conservation science scenario can be realized as media art, exhibiting the scenario immediately is impractical. Instead, through the living lab, we must conduct continuous on-site demonstrations and evaluation of user tests. In particular, we should prepare improvement plans to secure content diversity suitable for diverse visitors' ages and understanding and also disperse the bottleneck of experiential exhibitions.

\section{ACKNOWLEDGMENTS}

This research was supported by Scientific and Cultural Exhibition Service Program through the National Research Foundation of Korean (NRF) funded by the Ministry of Science, ICT (NRF-2018X1A3A1070017).

\section{REFERENCES}

Bai, J.S. and Boo, J.C., 2011, Study on museum digital exhibition mode and industrialization of intangible cultural heritage. International Journal of KIMICS, 9(2), 129-134. (in English)

Grundhöfer, A. and Iwai, D., 2018, Recent advances in projection mapping algorithms, hardware and applications. Computer Graphics Forum, 37(2), 653-675. (in English)

Guo, Y., Chu, S.C., Liu, Z., Qiu, C., Luo, H. and Tan, J., 2018, A real-time interactive system of surface reconstruction and dynamic projection mapping with RGB-depth sensor and projector. International Journal of Distributed Sensor Networks, 14(7), 1-12. (in English)

Jeon, H.T., 2016, A study of development exhibition contents for Korean petroglyphs digital museum. Humanities Contents, 43, 25-40. (in Korean with English abstract)

Jeon, I.H., Lee, J.S., Baek, J.H. and Park, J.S., 2008, Surface treatments of bronze mirrors excavated from Korean peninsula. Journal of Conservation Science, 22, 87-98. (in Korean with English abstract)

Kim, M., Sohn, Y. and Yoon, J., 2009, Research on experimental culture contents based on media art. Journal of Korea Design Forum, 22, 265-274. (in Korean with English abstract)

Krautsack, 2011, 3D projection mapping and its impact on media $\&$ architecture in contemporary and future urban spaces. Journal of the New Media Caucus, 7, 1. (in English)

Kuo, C., Lee, Y. and Wang M.C., 2016, The digital museum: a case study of digital applications in the national palace museum. Global Journal of Human-Social Science, 16, 30-38. (in English)

Nofal, E., Stevens, R., Coomans, T. and Moere, A.V., 2018, Communicating the spatiotemporal transformation of architectural heritage via an in-situ projection mapping installation. Digital Applications in Archaeology and Cultural Heritage, 11, e00083. (in English)

Noh, S.S., Lee, J.J., Kim, H.G. and Park, J.W., 2011, Interactive media art works using culture archetype. Journal of Korean Society of Design Science, 25(1), 157-166. (in Korean with English abstract)

Seong, M.J. and Lee, D.H., 2017, Design and implementation of cultural property learning contents using augmented reality. Journal of Digital Contents Society, 18(5), 831-837. (in Korean with English abstract)

Yamazoe, H., Kasetani, M., Noguchi, T. and Lee, J.H., 2018, Projection mapping onto multiple objects using a projector robot. Advances in Robotics Research, 2(1), 45-57. (in English)

Yun, H.R., Kim, D.W. and Ishii, T., 2013, A study of digital media art utilizing the contents of the architecture cultural property. International Journal of Asia Digital Art \& Design, 17(2), 77-84. (in English) 